- Sjögren's syndrome may lead to dental caries, periodontal disease, denture intolerance, candidosis and dysphagia.

- This study evaluated the use of a hydrogel polymer buccal insert as a controlled release delivery vehicle for pilocarpine.

- The insert delivered in excess of $85 \%$ of a $5 \mathrm{mg}$ dose of pilocarpine hydrochloride with minimal side-effects; oral and eye comfort scores generally improved on therapy.

\title{
A controlled release pilocarpine buccal insert in the treatment of Sjögren's syndrome
}

\author{
J. Gibson, ${ }^{1}$ J. A. Halliday, ${ }^{2}$ K. Ewert ${ }^{3}$ and S. Robertson ${ }^{4}$
}

\begin{abstract}
Objectives To assess the efficacy of a novel hydrogel polymer buccal insert containing $5 \mathrm{mg}$ pilocarpine in releasing the pilocarpine in a controlled fashion over a three hour period, and to assess the effects of this on quantitative tear and saliva production and the acceptability of the insert to the patient.

Design This was an open, uncontrolled pilot study for which Ethics Committee approval was obtained prior to starting. Hydrogel buccal inserts containing $5 \mathrm{mg}$ pilocarpine were used three times a day for seven days.
\end{abstract}

Setting The Department of Oral Medicine, Glasgow Dental Hospital et School.

Subjects Eight patients with Sjögren's syndrome.

Main outcome measures Changes over baseline in (1) Schirmer test, (2) whole saliva flow rate, (3) oral comfort score (VAS), (4) ocular comfort score (VAS), (5) patient acceptability.

Results The buccal inserts successfully released in excess of $85 \%$ of their $5 \mathrm{mg}$ pilocarpine load over three hours. There was a general improvement in oral and ocular comfort scores assessed by visual linear analogue scale, and saliva and tear production generally increased. The inserts were well tolerated by all patients except one (who wore dentures). Adverse events were few and none was serious.

Conclusion This novel form of buccal pilocarpine delivery demonstrated potential for use in treating patients with Sjögren's syndrome.

\section{INTRODUCTION}

Sjögren's syndrome (SS) is a chronic autoimmune condition characterised by infiltration of lacrimal and salivary glands by lymphocytes, leading to dryness of the mouth (xerostomia) and/or dryness of the eyes (xerophthalmia). ${ }^{1}$ The syndrome is

\footnotetext{
${ }_{1}^{*}$ Consultant and Honorary Senior Lecturer in Oral Medicine, Dundee Dental Hospital \&t School, Park Place, Dundee, DD1 4HN; ${ }^{2}$ Director of Research and Development,

${ }^{3}$ Clinical Project Manager, ${ }^{4}$ Director of Strategic Projects, Controlled Therapeutics Ltd,

1 Redwood Place, Peel Park Campus, East Kilbride, G74 5PB

${ }^{*}$ Correspondence to: Dr John Gibson

Email:john.gibson2@nhs.net
}

Online article number E17

Refereed Paper - accepted 18 April 2006

DOI: $10.1038 /$ bdj.2007.80

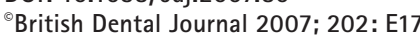

estimated to affect 500,000 or more people in the UK. ${ }^{2}$ The disease may present secondary to other autoimmune diseases, such as rheumatoid arthritis and systemic lupus erythematosus, but it is also recognised as presenting without any other concurrent autoimmune condition: it is then termed primary Sjögren's syndrome. It is a disabling condition and may lead to severe dental caries, periodontal disease, denture intolerance, candidosis and dysphagia. Patients consequently require regular dental care and supervision. Treatment is normally palliative, involving mouthwashes, toothpastes, chewing gums and saliva substitutes. A variety of herbal and pseudo-pharmaceutical products is also available. ${ }^{3}$ The drug cevimeline hydrochloride (a muscarinic M1 and M3 receptor agonist) has been approved by the FDA (Evoxac ${ }^{\circledR}$, Daiichi Pharmaceutical Co. Ltd) for the treatment of xerostomia in patients with Sjögren's syndrome in the USA. ${ }^{4}$ Cevimeline has a greater specificity for M3 receptors than pilocarpine but it is not available in the UK. There is, therefore, a need for another product which will minimise side effects and alleviate the symptoms of xerostomia. The modern understanding of the salivary hypofunction in Sjögren's syndrome is that it is thought to be the result of glandular inhibition. Therefore, any attempt to stimulate flow is to be encouraged as it is likely to maintain salivary gland mass. ${ }^{5}$ This drug has shown promise in various studies as a potentially valid treatment for xerostomia. However, the sideeffect profile may limit clinical use. ${ }^{6}$

In recent years the muscarinic agonist pilocarpine (Salagen ${ }^{\circledR}$, Novartis) has been used to some effect. ${ }^{7}$ It was launched in the UK in 1995, originally only for xerostomia associated as a side effect of radiotherapy to treat head and neck cancer; as a normal release oral tablet it delivers $5 \mathrm{mg}$ of pilocarpine hydrochloride. When taken three or four times daily in doses of 5-10 $\mathrm{mg}$, it is capable of increasing both salivary flow and lacrimal secretions. The tablet is normally taken with food to counteract some of the more obvious and common adverse reactions of the gastro-intestinal tract. It may cause a variety of side effects including sweating, nausea, diarrhoea, dyspepsia, abdominal pain, flushing, headache, dizziness, rhinitis, 
increased urinary frequency, influenza-like syndrome and, less frequently, vomiting, flatulence, constipation, palpitations, hypertension, urinary urgency, blurred and altered vision. ${ }^{3}$ This side effect profile may cause some patients to become disillusioned with therapy and to revert to the standard palliative regime.

A study by Lockhart et al. ${ }^{8}$ examined a controlled-release pilocarpine preparation in the treatment of xerostomia in Sjögren's syndrome and this showed some promise. It was thus considered that a controlled release delivery method may reduce the amount of drug that is absorbed systemically and thus reduce the incidence of side effects. Hydrogels are widely used as contact lens materials and have found application in the biomedical field and in drug delivery.

The aim of this study was to evaluate the use of a hydrogel polymer buccal insert as a controlled release delivery vehicle for pilocarpine. The insert used was an adaptation of one used in marketed products as a vaginal delivery system for dinoprostone in childbirth (Cervidil ${ }^{\oplus}$, Propess ${ }^{\circledast}$ ) and for rectal morphine for control of severe pain $\left(\right.$ Moraxen $\left.^{\circ}\right){ }^{9}$ A small uncontrolled study previously carried out in six healthy volunteers using buccal inserts $1 \mathrm{~mm}$ in thickness had indicated that the inserts (without any medication) were well tolerated: the only adverse events were changes in colour to the mucosal surfaces after three hours, and these resolved upon removal of the insert. Further laboratory work indicated that a reduction in the thickness of the insert would provide more effective drug delivery when administered by the buccal route. The objectives of this study were to assess the effectiveness of a $0.6 \mathrm{~mm}$ thick hydrogel buccal insert containing $5 \mathrm{mg}$ pilocarpine hydrochloride in providing the controlled release of pilocarpine over a three-hour period, the effects of pilocarpine on quantitative tear and saliva production and the acceptability of the insert to the patient.

\section{MATERIALS AND METHODS}

\section{Study design and setting}

This open, uncontrolled pilot study took place in the Department of Oral Medicine, Glasgow Dental Hospital and School, according to ICH GCP Guidelines, over a period of 14 days. The study drug was provided by the hydrogel manufacturer, Controlled Therapeutics (Scotland) Ltd. After the protocol was reviewed and approved by the Area Ethics Committee, patients were recruited from those attending for review of their Sjögren's syndrome.

\section{Patients}

All enrolled patients gave written, informed consent prior to the start of the study. To be included in the study a patient had to have a confirmed diagnosis of Sjögren's syndrome. Patients with a condition for which pilocarpine was contraindicated, who had uncontrolled asthma or chronic obstructive airways, active cardiovascular or peptic ulcer disease, cholelithiasis, renal or hepatic impairment, psychiatric disturbance or took concomitant anti-muscarinic or beta-blocker drugs were excluded. Of eight patients recruited (six female and two male), six had primary and two had secondary Sjögren's syndrome as defined by the modified European classification criteria..$^{10}$ These criteria were later revised in 2002 and following retrospective review of the data, only one patient

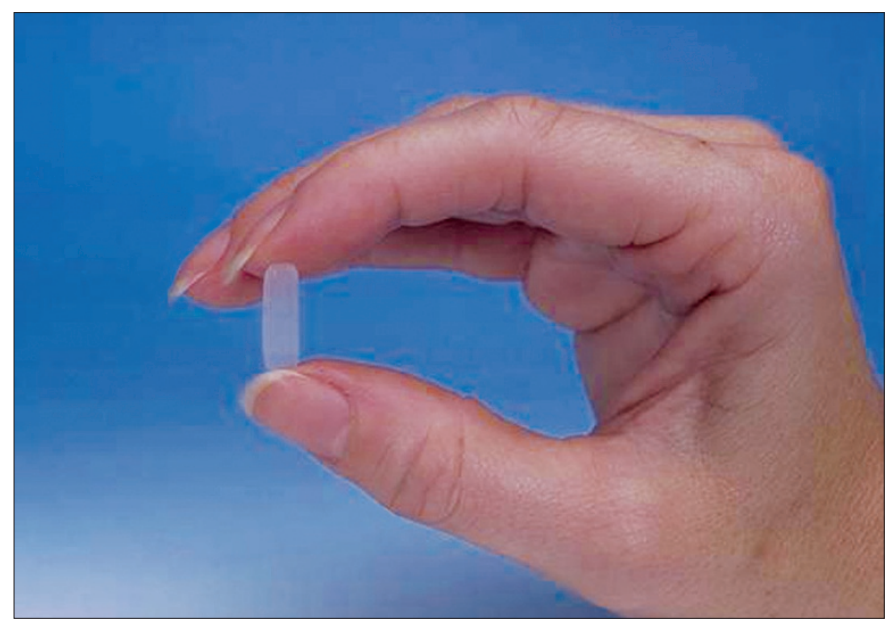

Fig. 1 Hydrogel buccal insert showing shape and size

(patient three) did not meet the requirements of the revised international classification criteria for Sjögren's syndrome. ${ }^{11}$ Prior to the start of the study, an ophthalmologist assessed all patients to ensure there were no ophthalmic contra-indications to the use of pilocarpine, and all patients were counselled regarding possible visual disturbances during pilocarpine administration. Pilocarpine is contra-indicated in certain ophthalmic conditions such as acute iritis, anterior uveitis and some types of glaucoma.

\section{Hydrogel polymer buccal insert}

The hydrogel polymer buccal inserts used in this study were $17 \mathrm{~mm}$ in length, $5 \mathrm{~mm}$ wide and $0.6 \mathrm{~mm}$ thick (Fig. 1). The ends were rounded for comfort and the inserts could be placed unobtrusively into the buccal sulcus.

The insert is non-biodegradable and functions by swelling in the presence of moisture and releasing pilocarpine in a controlled manner into the mouth for local absorption into the systemic circulation. As the insert swells in contact with saliva, it becomes softer and more pliable, but it does not dissolve. When placed in the sulcus, the insert adheres to the mucous membrane and stays in place for up to three hours. Adhesion is due to the muco-adhesive properties physically related to the swelling of the hydrogel polymer, and no additional adhesives or glues are required. The period for which the insert remains in place is related to several factors, but principally the hydration status of the mouth. Once detached, the insert may either be removed or returned to its former position, or a new position, within the mouth to continue to release drug. In this study the inserts contained $5 \mathrm{mg}$ of pilocarpine hydrochloride.

\section{Study procedures}

The study lasted 14 days, with therapy starting on day eight. On day one, patients were screened and any enrolled patient using pilocarpine had this therapy withdrawn for a seven day washout period. Based on the pharmacological literature, it was anticipated that a seven day washout period would be sufficient for all residual pilocarpine to be eliminated from the body.

Prior to administering the first dose, patients were shown how to place the insert high in the upper buccal sulcus and they were supplied with sufficient inserts containing 
$5 \mathrm{mg}$ pilocarpine for seven days. On days 8-14, patients were asked to use one insert three times per day, keeping each insert in place for three hours. They were also asked to record any adverse events whilst taking the study medication.

\section{Clinical assessments}

On day one, baseline measurements (with no insert in place) were made of lacrimal flow rates using Schirmer test strips on both eyes over five minutes, ${ }^{10}$ and salivary flow rates were measured by recording the volume of unstimulated saliva produced into a Sterilin tube over a 15 minute period..$^{10}$ Patients were asked to record their own assessment of symptoms of dry mouth and eyes using a linear visual analogue scale (VAS) that ranged from 0 (most comfortable) to 10 (most uncomfortable). On days eight, 10 and 12 patients were asked to attend the clinic to have a Schirmer test carried out (once per day, in the morning, while the insert was in place) and salivary flow rates measured (as per day one). All clinical assessments were carried out on all occasions by the same investigator (JG) to avoid inter-observer variation.

\section{Analysis of used inserts}

At the end of each period of insertion, the patient placed the used insert into a ziplock bag and stored it in the freezer. The

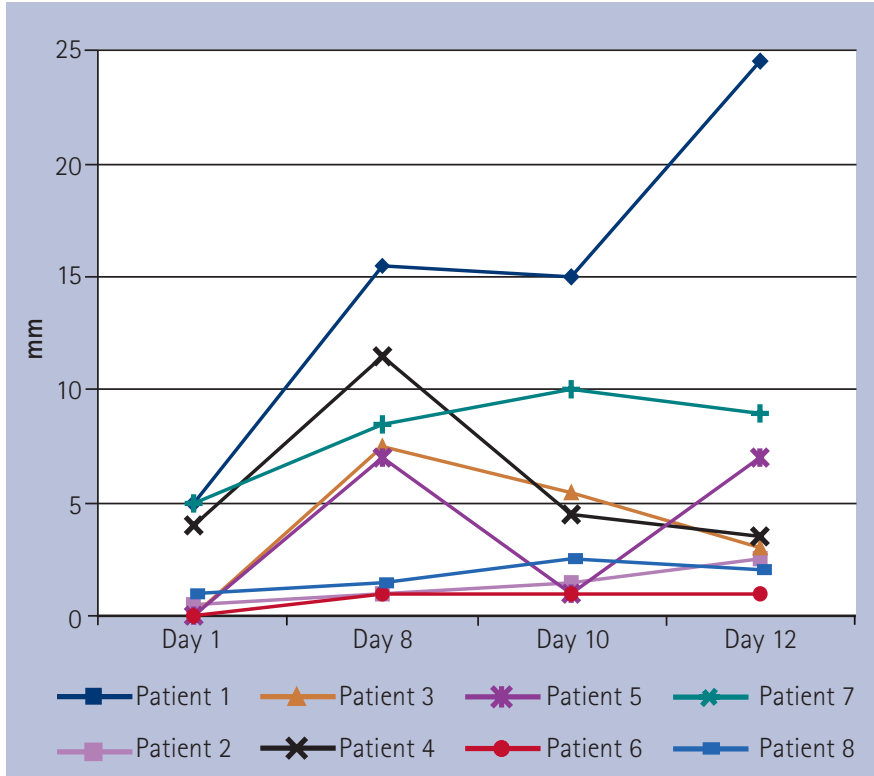

Fig. 2 Schirmer test results (average for both eyes) used inserts were collected and maintained at $-20^{\circ} \mathrm{C}$ until laboratory analysis. Laboratory analysis was carried out by Controlled Therapeutics (Scotland) Ltd in the company's premises at East Kilbride, using high performance liquid chromatography (HPLC). The analysis detected the amount of pilocarpine remaining in the used inserts. This result was then used to calculate the amount of drug released from the insert over the dosing period. This calculation was measured by subtracting the amount of pilocarpine remaining in the used insert from the mean amount of drug from all the inserts made prior to the start of the study (4.8 $\mathrm{mg})$.

\section{RESULTS}

\section{Patient population}

The characteristics of study patients are shown in Table 1. Patients had a mean age of 60 years (range 49-70) and all had had a confirmed diagnosis of SS for at least three years. All except one had previously used pilocarpine but only patients one, two, five and seven were using pilocarpine preparations immediately prior to the wash-out phase.

\section{Clinical assessments}

Results were statistically analysed using the Wilcoxon signed ranks test. In each of the tables and graphs, day one = baseline results; days eight, 10,12 and 14 are representative dosing days.

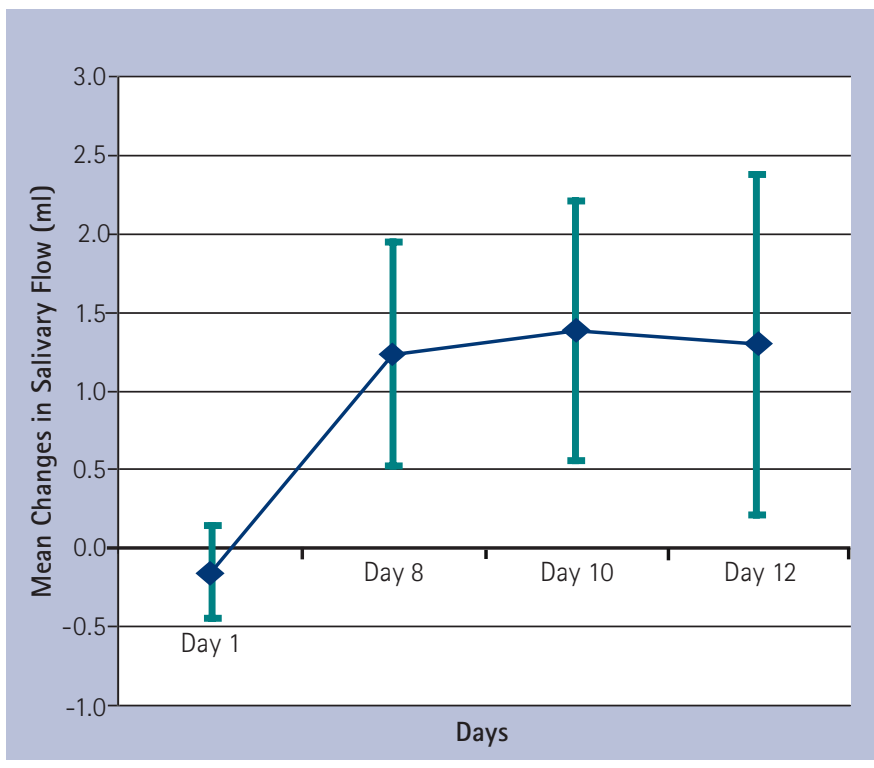

Fig. 3 Mean change in salivary flow rates (whole unstimulated flow; $\mathrm{ml}$ ) (days 1-7 pre-dosing, days 8-14 dosing)

Table 1 Demography of study patients

\begin{tabular}{l|l|l|l|l|l|l|l}
\hline Patient & Age & Sex & Race & Primary or secondary SS & Associated disorder & Duration of SS (years) & Prior pilocarpine use? \\
\hline 1 & 63 & F & Caucasian & primary & - & 5 & yes \\
\hline 2 & 51 & F & Caucasian & secondary & Lupus (SLE) & 3 & yes \\
\hline 3 & 65 & M & Caucasian & primary & - & 4 & no \\
\hline 4 & 53 & M & Caucasian & primary & - & 4 & yes \\
\hline 5 & 49 & F & Caucasian & primary & - & 3 & yes \\
\hline 6 & 70 & F & Caucasian & primary & - & 4 & yes \\
\hline 7 & 59 & F & Caucasian & primary & - & 3 & yes \\
\hline 8 & 63 & F & Caucasian & secondary & Lupus (SLE) & 7 & yes \\
\hline
\end{tabular}




\begin{tabular}{|c|c|c|c|c|c|c|c|}
\hline \multirow[t]{5}{*}{ Mean } & \multirow[t]{2}{*}{ Day } & \multicolumn{6}{|c|}{$\begin{array}{l}\text { Amount of pilocarpine released }(\mathrm{mg}) \text { and as } \\
\% \text { of } 5 \mathrm{mg} \text { dose }\end{array}$} \\
\hline & & \multicolumn{2}{|c|}{ Morning } & \multicolumn{2}{|c|}{ Afternoon } & \multicolumn{2}{|c|}{ Evening } \\
\hline & 8 & 4.6 & $96 \%$ & 4.5 & $94 \%$ & 4.1 & $85 \%$ \\
\hline & 10 & 4.6 & $96 \%$ & 4.6 & $96 \%$ & 4.3 & $90 \%$ \\
\hline & 14 & 4.8 & $100 \%$ & 4.4 & $92 \%$ & 4.3 & $90 \%$ \\
\hline \multirow[t]{5}{*}{ Median } & Day & \multicolumn{6}{|c|}{$\begin{array}{l}\text { Amount of pilocarpine released }(\mathrm{mg}) \text { and as } \\
\% \text { of } 5 \mathrm{mg} \text { dose }\end{array}$} \\
\hline & & \multicolumn{2}{|c|}{ Morning } & \multicolumn{2}{|c|}{ Afternoon } & \multicolumn{2}{|c|}{ Evening } \\
\hline & 8 & 4.8 & $100 \%$ & 4.6 & $96 \%$ & 4.5 & $94 \%$ \\
\hline & 10 & 4.8 & $100 \%$ & 4.8 & $100 \%$ & 4.6 & $96 \%$ \\
\hline & 14 & 4.8 & $100 \%$ & 4.5 & $94 \%$ & 4.7 & $98 \%$ \\
\hline \multirow[t]{5}{*}{ Range } & Day & \multicolumn{6}{|c|}{$\begin{array}{l}\text { Amount of pilocarpine released }(\mathrm{mg}) \text { from origina } \\
5 \mathrm{mg} \text { dose }\end{array}$} \\
\hline & & \multicolumn{2}{|c|}{ Morning } & \multicolumn{2}{|c|}{ Afternoon } & \multicolumn{2}{|c|}{ Evening } \\
\hline & 8 & 3.9 & 4.8 & 3.8 & 4.8 & 2.6 & 4.8 \\
\hline & 10 & 3.5 & 4.8 & 3.5 & 4.8 & 2.9 & 4.8 \\
\hline & 14 & 4.6 & 4.8 & 3.9 & 4.8 & 3.2 & 4.8 \\
\hline
\end{tabular}

For the range of release, the first column shows the lowest amount and the second column shows the highest amount for each insert

Table 3 Schirmer test results (left eye/right eye; measurements in
mm; day 1 is pre-dosing; days 8,10 and 12 are dosing days)
\begin{tabular}{l|l|l|l|l|l|l|l|l|}
\hline \multirow{2}{*}{ Day } & Patient number \\
\cline { 2 - 9 } & 1 & 2 & 3 & 4 & 5 & 6 & 7 & 8 \\
\hline 1 & $5 / 5$ & $1 / 0$ & $1 / 2$ & $3 / 5$ & $3 / 0$ & $0 / 0$ & $5 / 5$ & $1 / 1$ \\
\hline 8 & $16 / 15$ & $1 / 1$ & $3 / 12$ & $10 / 13$ & $10 / 4$ & $1 / 1$ & $7 / 10^{*}$ & $2 / 1$ \\
\hline 10 & $15 / 15$ & $1 / 2$ & $5 / 6$ & $4 / 5$ & $2 / 0^{*}$ & $1 / 1$ & $10 / 10^{*}$ & $3 / 2$ \\
\hline 12 & $25 / 24$ & $3 / 2$ & $3 / 3$ & $4 / 3$ & $8 / 6$ & $1 / 1$ & $8 / 10^{* *}$ & $2 / 2$ \\
\hline
\end{tabular}

Day 1: no insert in place; days 8, 10 and 12: insert in place unless otherwise specified.

*Test conducted without insert in place.

**Unknown if insert in place during test procedure.

\section{Drug release characteristics of hydrogel insert}

Table 2 shows the mean, median and range of the quantity of drug released for the eight patients on days eight, 10 and 14. This drug quantity was calculated by subtracting the amount of pilocarpine remaining in the used insert from the mean amount of drug previously determined from analysis of all the inserts made prior to study start ( $4.8 \mathrm{mg}$ ). It can be seen that the inserts released almost all of the dose of pilocarpine during the three hour dosing period.

\section{Schirmer test}

This test was carried out over five minutes in general between 59 and 135 minutes following the insertion of the buccal insert. The results are presented in Table 3 for each eye and in Figure 2 for the average of both eyes, and are indicative of the diverse nature of this group of patients. On three occasions, patients undertook the Schirmer test without the insert in place: patient five on day 10 and patient seven on days eight and 10. On day 12 it was unrecorded whether patient seven had the insert in place or not.

\begin{tabular}{l|l} 
Table 4 & Adverse events recorded by patients during the study \\
\hline Patient & Comments \\
\hline 1 & $\begin{array}{l}\text { Slight discomfort in mouth (after dose) } \\
\text { Mild erythema in the upper left buccal sulcus }\end{array}$ \\
\hline 2 & Mild flushing (25 minutes after insertion) \\
\hline 3 & $\begin{array}{l}\text { Slight increase in sweating } \\
\text { Increased pulse rate } \\
\text { Throat infection, day 2 (did not take morning dose) }\end{array}$ \\
\hline 4 & $\begin{array}{l}\text { Mild headache } \\
\text { Dry lips } \\
\text { Dryeyes } \\
\text { Sweating during the night } \\
\text { 'Fuzzy head' }\end{array}$ \\
\hline 5 & $\begin{array}{l}\text { Terrible taste from insert } \\
\text { Slight tingling on upper lip on side of insert } \\
\text { Throbbing of carotid pulse } \\
\text { Tingling tongue } \\
\text { Drying of mouth } \\
\text { Slightly swollen tongue } \\
\text { Gingiva - slightly raw feeling on side of insert } \\
\text { Very loose stool (x4 morning, x1 evening) } \\
\text { Sore throat on side of insert } \\
\text { Tongue uncomfortable - worse at night } \\
\text { Surface of palate raw } \\
\text { Pain between eyes }\end{array}$ \\
\hline 7 & \begin{tabular}{l} 
None \\
\hline 8
\end{tabular} \\
\hline $\begin{array}{l}\text { Mild stomach pain (during insertion) } \\
\text { Gum discomfort at site of insert } \\
\text { Shivering/sweating (during insertion) } \\
\text { Increased salivation } \\
\text { Headache } \\
\text { Dry throat } \\
\text { Ulceration (caused by dentures) }\end{array}$ \\
\hline None \\
\hline 6
\end{tabular}

The results presented suggest that there is an increase in left/right eye measurement at day 12 when compared to day one ( $p=0.0156)$; an increase in tear production is shown by increase in Schirmer measurement.

The half-life of the pilocarpine buccal insert was not known during the conduct of this study, therefore the collection times were not standardised. However, a pharmacokinetic study was later conducted in healthy volunteers and results showed that the half-life of the insert containing $10 \mathrm{mg}$ of pilocarpine was approximately 1.5 hours (unpublished data).

\section{Salivary flow rate}

The mean change in salivary flow rates taken over a 15 minute period after dosing are shown in Figure 3. Salivary measurements were taken between one and five minutes after the completion of the Schirmer test but on day eight, patients six and eight each carried out the salivary flow test before the Schirmer test.

There is a marked improvement in the mean change in salivary flow rate at day eight compared to day one values, ie increase from 0 to $1.2 \mathrm{ml}$. This new elevated flow rate is sustained for a further four days.

The results presented suggest that there is an increase in salivary flow in patients at day 12 when compared to day one $(\mathrm{p}=0.0078)$.

\section{VAS oral comfort scores}

Figure 4 presents the percentage change in the summated VAS 


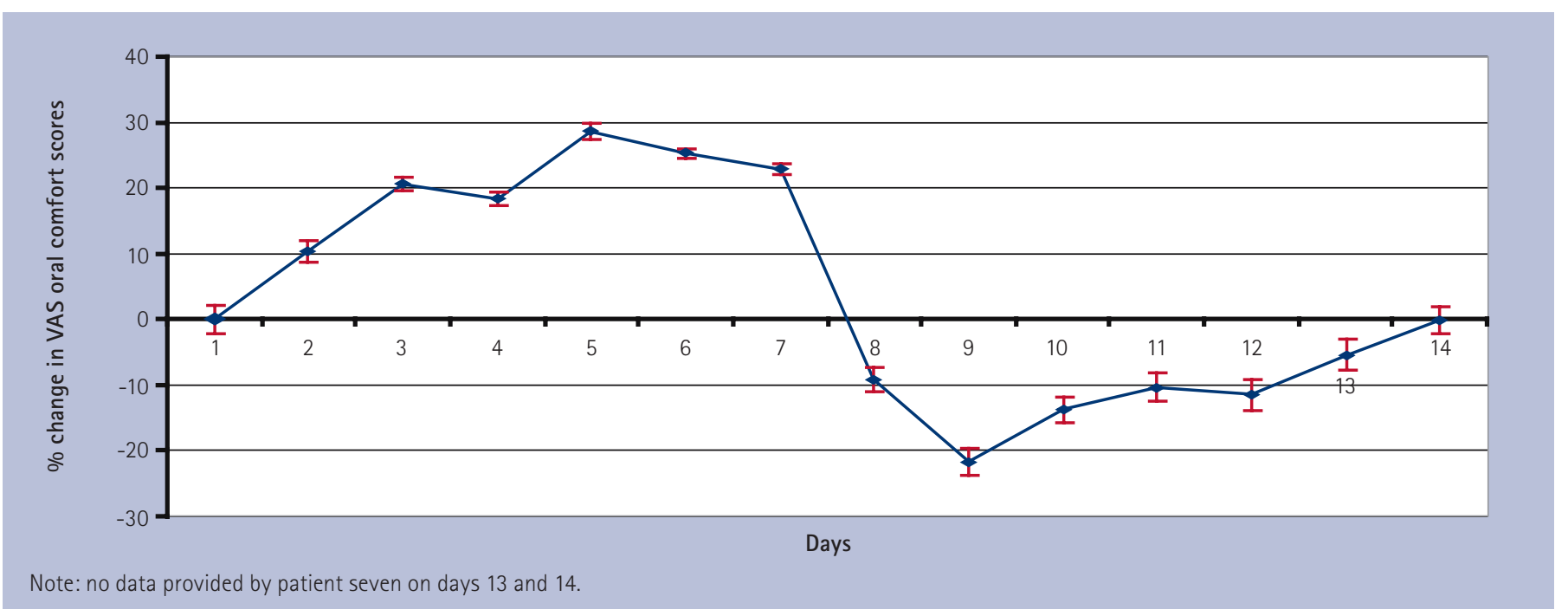

Fig. 4 Percentage change in VAS oral comfort scores (days 1-7 pre-dosing, days 8-14 dosing)

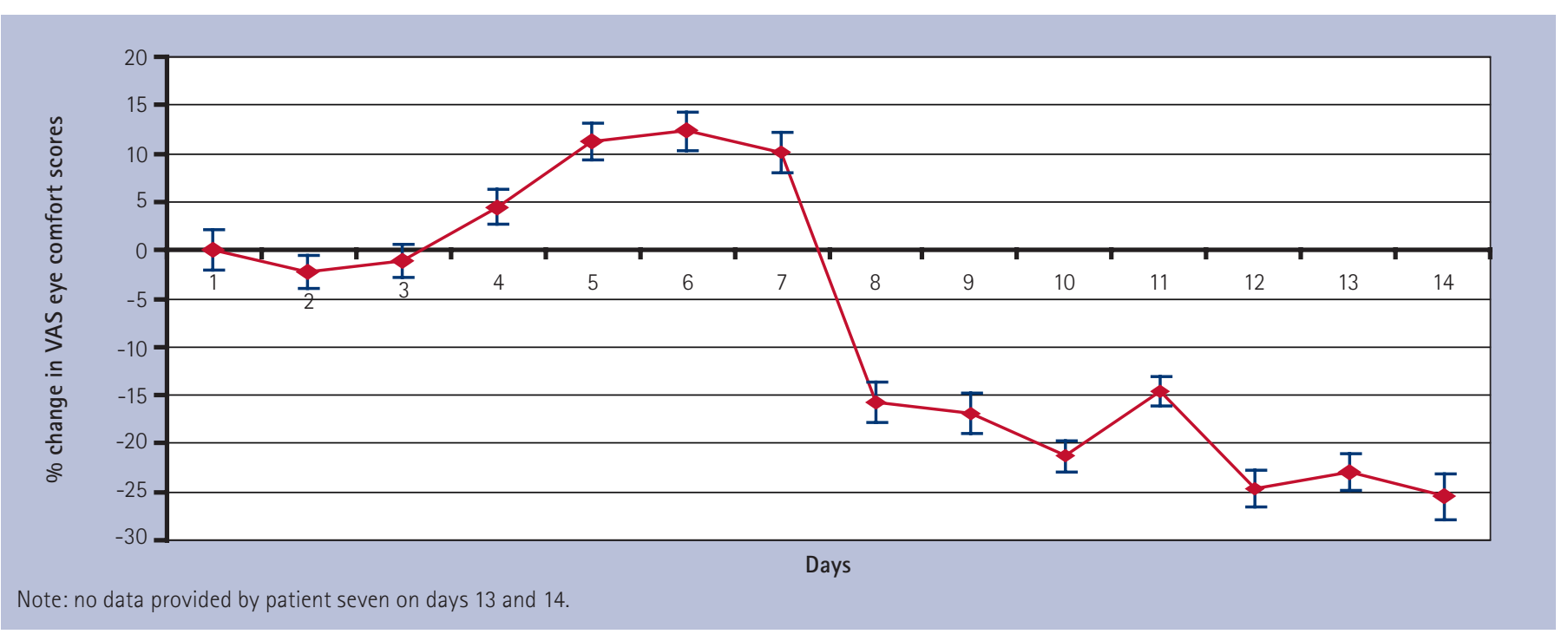

Fig. 5 Percentage change in VAS eye comfort scores (days 1-7 pre-dosing, days 8-14 dosing)

oral comfort scores. The patients underwent a seven day washout period prior to the first dosing day (day eight). The figure shows that after day two of the washout period, a percentage increase of $20-30 \%$ is sustained up to day seven. This correlates with decreasing comfort for the patients. With onset of treatment at day eight, an immediate marked improvement in VAS oral comfort score was observed. The figure shows a 10$20 \%$ reduction from baseline between days eight and 12 .

The data suggests that there is a difference between the VAS oral comfort score data collected at day seven and day 12 ( $\mathrm{p}=$ 0.0469). The results suggest that the values on day 12 are lower than those collected on day seven, which shows an improvement in comfort.

\section{VAS eye comfort scores}

Figure 5 shows the percentage change in the summated VAS scores for eye comfort. These results support the VAS oral comfort scores (Fig. 4). A marked improvement in eye comfort scores is observed following treatment with the buccal inserts from day eight.

The data suggests that there is a difference between the VAS eye comfort score data, where there is a lower value on day
12 when compared to day seven, suggesting better comfort $(\mathrm{p}=0.0156)$.

\section{Adverse events}

During the course of the study a number of adverse events were observed, including abdominal discomfort, flushing, sweating and headache. All of these are known side-effects of pilocarpine administration. In addition, two patients reported an unusual taste and sore or dry throat. Local mucosal erythema (with some discomfort reported) was observed in patient one at the site of insert placement. Side-effects, where reported, were generally recorded as most obvious on day 10 of the study. Patient seven withdrew from the study after day 12 due to localised oral ulceration because of the presence of a complete upper denture and the resulting rubbing of the insert on the adjacent buccal mucosa. The adverse events recorded during the study are shown in Table 4.

\section{DISCUSSION}

Recent research into the use of the polymer as a bio-adhesive polymer delivery system for the mouth has led to this possible application in Sjögren's syndrome. The lack of salivary 
flow in this group of patients might, at first, suggest that the insert would be ineffective. However, this study has shown that the insert will swell and release the drug effectively over a three hour period in patients with this condition, apparently drawing moisture from the mucosa to facilitate swelling. There were noticeable improvements at the time of first administration of the pilocarpine inserts in oral and eye comfort scores, and increases in salivary and lacrimal secretions in the majority of patients.

These improvements over baseline were generally maintained below peak levels to the end of dosing, although there was considerable variation in the responses to the pilocarpine. However, the severity of Sjögren's syndrome can vary widely between patients and in the most advanced stages, the patient may have little or no functional salivary tissue left. The degree to which pilocarpine has a beneficial effect is dependent upon the extent of the disease, however administered, and this may explain some of the variability of the Schirmer test between patients. Variability may also have been affected by the fact that not all patients were consistent in the timing of the Schirmer test. Although most were undertaken between 59 and 135 minutes after the buccal insert was in place, on $12.5 \%$ of occasions the buccal insert was not in place, and for a further $4.2 \%$ it is not known whether the insert was in place or not. However, day one records show almost all the patients to have very little lacrimal secretion in the absence of therapy, while days eight, 10 and 12 show improvement in most cases.

None of the adverse events reported were unexpected. What is not known is whether the rate of drug release was constant, or whether most of the drug was released at some point before the end of the three hour period. Further work is planned to investigate these factors and to examine whether improvements can be made to the design and shape of the hydrogel insert.

The study design was limited, as additional data could have been obtained to suggest how effective the pilocarpine buccal inserts were compared to oral pilocarpine treatment and no treatment at all. Larger controlled studies are required in order to statistically determine the effectiveness of the pilocarpine buccal insert.

\section{CONCLUSIONS}

This novel use of a pilocarpine-containing hydrogel polymer insert in patients with Sjögren's syndrome has shown that it effectively and conveniently delivered in excess of $85 \%$ of a $5 \mathrm{mg}$ dose of pilocarpine hydrochloride with minimal adverse events. There appeared to be little inhibition of swelling of the hydrogel because of the underlying Sjögren's syndrome, except in one patient where the disease was very advanced. Drug release was excellent over the three hour period and oral and eye comfort generally improved on therapy. Further work, which will include placebo inserts, will establish the thickness required to give optimal controlled release.

This hydrogel polymer insert offers a promising method of delivering pilocarpine in a controlled way for the management of patients with Sjögren's syndrome, to maximise benefit and minimise side-effects.

\section{Competing interests}

This study drug was provided by Controlled Therapeutics (Scotland) Ltd. $J G$ was the Principal Investigator and was involved in all stages of the study except for the manufacture of the inserts and the analysis of the used inserts. These tasks were undertaken by Controlled Therapeutics (Scotland) Ltd. The study design, conduct of the study, collection of data and analysis of results have complied with pharmaceutical industry guidelines. Writing of this paper was led by JG with input from JAH, KE and SR. Given the involvement of three employees of Controlled Therapeutics (Scotland) Ltd (JAH, KE and SR) in the development of the overall pilocarpine buccal insert project, it was the desire of JG and JAH, KE and SR that this involvement should be completely transparent and evident to the editor, referees and readership - hence the decision to include the names of JAH, KE and $S R$ in the authorship. We felt, together, that this was the most appropriate way to proceed in submitting this work for publication. JAH, KE and SR, by agreeing to co-authorship, have indicated their recognition of all potential conflicts of interest, their acceptance of all inherent professional responsibilities as pharmacists, registered nurses and scientists with regard to integrity, and willingly state that there has been no compromise at any stage in the scientific integrity of this paper.

1. Jonsson J, Moen K, Vestrheim D, Szodoray P. Current issues in Sjogren's syndrome. Oral Dis 2002; 8: 130-140.

2. Griffiths I. The enigma called Sjogren's Syndrome. Arthritis Today 2004; 123: 13-15.

3. Ship J A. Diagnosing, managing, and preventing salivary gland disorders. Oral Dis 2002; 8: 77-89.

4. Fox R I. Use of cevimeline, a muscarinic $\mathrm{M} 1$ and $\mathrm{M} 3$ agonist, in the treatment of Sjogren's syndrome. Adv Exp Med Biol 2002; 506: 1107-1116.

5. Dawson L J, Smith P M, Moots R J, Field E A. Sjögren's syndrome - time for a new approach. Rheumatology 2000; 39: 234-237.

6. Fife R S, Chase W F, Dore R K et al. Cevimeline for the treatment of xerostomia in patients with Sjögren's syndrome: a randomised trial. Arch Intern Med 2002; 162: 1293-1300.

7. Vivino F B, Al Hashimi I, Khan Z et al. Pilocarpine tablets for the treatment of dry mouth and dry eye symptoms in patients with Sjögren's syndrome: a randomized, placebo-controlled, fixed-dose, multicenter trial. P92-01 Study Group. Arch Intern Med 1999; 159: 174-181.

8. Lockhart P B, Fox P C, Gentry A C, Acharya R, Norton H J. Pilot study of controlled release pilocarpine in normal subjects. Oral Surg Oral Med Oral Pathol Oral Radiol Endod 1996; 82: 517-524.

9. Graham N B, McNeill M E. Hydrogels for controlled drug delivery. Biomaterials 1984: $5: 27-35$

10. Vitali C, Bombardieri S, Moutsopoulos H M et al. Assessment of the European classification criteria for Sjögren's syndrome in a series of clinically defined cases: results of a prospective multicentre study. The European study group on diagnostic criteria for Sjögren's syndrome. Ann Rheum Dis 1996; 55: 116-121.

11. Vitali C, Bombardieri S, Jonsson R et al. Classification criteria for Sjögren's syndrome: a revised version of the European criteria proposed by the AmericanEuropean Consensus Group. Ann Rheum Dis 2002; 61: 554-558. 